\title{
Innovación tecnológica en la industria automovilística catalana
}

\author{
Technological innovation catalan in the automotive industry
}

\author{
Francisco Llorente Galera \\ Departamento de Econometría, Estadística y E. E. Facultad de Ciencias Económicas y Empresariales. Universidad de Barcelona \\ florente@ub.edu
}

\begin{abstract}
Resumen. El presente artículo presenta los resultados de un estudio para una muestra de empresas proveedoras directas de los fabricantes, segmentadas según la nacionalidad del capital o el tamaño empresarial, para saber si realizan innovaciones tecnológicas, qué personal disponen en $\mathrm{I}+\mathrm{D}+\mathrm{i}$, y si utilizan diferentes sistemas y técnicas que reducen el tiempo y costes de desarrollo de sus productos. Se verifica que son pocas las que utilizan tales sistemas y técnicas conjuntamente, siendo las grandes empresas las que más realizan I+D+i e incorporan tales sistemas y técnicas, al contrario que las pequeñas.
\end{abstract}

Palabras clave: automóvil, proveedores, innovación tecnológica, técnicas, I+D, Cataluña

Abstract: This paper offers the results of a study with a sample of direct suppliers firms of OEM, distinguished by capital nationality or firms size, to know if they make technological innovation, which R\&D\&l personnel they have and if they use different systems and techniques that reduce the time and costs of their products development. There are few firms that use these systems and techniques, so the greatest ones make more R\&D\&I and use these systems and techniques, unlike the small ones.

Key words: Car, suppliers, technological innovation, techniques, research and development, Catalonia.

\section{Introducción}

El sector automovilístico está inmerso en importantes cambios en los últimos años (globalización, exceso de capacidad, concentración y consolidación bajo una estructura piramidal, elevada competencia, deslocalización hacia países de menor coste, personalización del producto, incremento del lanzamiento de nuevos modelos y reducción de su ciclo de vida, etc.), necesitando satisfacer a unos clientes más exigentes, que desean más introducción de innovaciones tecnológicas (Wognum et al., 2002).

En un entorno tan competitivo con elevados ratios de renovación de los modelos la innovación es crucial (Aggeri y Segrestin, 2007) y obtener nuevos productos suelen ser clave para obtener éxito en el mercado (Miranda y Bañeguil, 2002), contribuyendo a mejorar las ventas y los beneficios (Koufteros et al., 2005). El automóvil es un producto complejo, implicando que el fabricante no puede disponer del adecuado know how del conjunto de tecnologías que incorpora, ni asumir los elevados riesgos y gastos de la I+D+i asociados a su interiorización en los nuevos modelos. Se queda con lo que considera sus capacidades nucleares (Hamel y Prahalad, 1994) y delega más actividades de I+D+i a un inferior número de proveedores, con capacidad tecnológica y productiva de ofrecerle adecuados precios, calidad y servicio (Laming, 1993; Anderson et al., 2000).
Los proveedores de los fabricantes necesitan ofrecer productos con más valor añadido, tendiendo a incorporar multitecnología en los mismos (p.e. integrar la mecánica y la electrónica con la mecatrónica o bien desarrollar materiales híbridos a partir de los metales y plásticos que dan materiales con nuevas propiedades y son más ligeros). Según VDA (2009) los fabricantes quieren que los proveedores se integren más en el desarrollo de los nuevos vehículos y se distingan con su innovación, para tener oportunidades de crecimiento. Tales proveedores deben ajustarse a las reducciones de tiempo de diseño y desarrollo que establecen los fabricantes para sus nuevos modelos y ajustar sus costes a los menores precios que les exigen. Ante ello precisan diseñar y desarrollar nuevos productos con alta calidad, en menor tiempo y costes inferiores, utilizando diversas técnicas y sistemas (Bañeguil, 200 I; Martínez y Pérez, 2003a,b), tendiendo a adoptar los principios de la empresa ajustada (Womack et al., 1990).

Las empresas que logran desarrollar sus nuevos productos en menos tiempo deberían obtener ventajas competitivas, si bien no se ha podido constatar de forma significativa en la literatura empresarial (Bañeguil y Miranda, 200 I; Langerak y Hulink, 2006).

En el presente artículo se desea conocer si los proveedores de los fabricantes de automóviles realizan innovaciones tecnológicas, efectúan actividades de 
investigación, diseño y desarrollo de producto, el personal asignado a I+D+i, y si utilizan ciertas técnicas y sistemas, que según la literatura reducen los costes, el tiempo de diseño y desarrollo, así como mejorar la calidad en el diseño y desarrollo de los nuevos productos. En concreto se han seleccionado las siguientes: Ingeniería Concurrente, CAD (diseño asistido por ordenador), CAM (fabricación asistida por ordenador), CAE (ingeniería asistida por ordenador), QFD (Desarrollo de la función de Calidad), AMFE (Análisis Modal de Fallos y Efectos), DFMA (Diseño para la fabricación y ensamblaje), Prototipado rápido, Ingeniería del valor, Análisis valor, Métodos Taguchi/DOE, Colaborar con clientes y proveedores.

A nivel empírico se presentan y analizan los resultados de una encuesta realizada a proveedores directos de los fabricantes de automóviles ubicados en Cataluña, segmentando por nacionalidad del capital y por tamaño empresarial ', para conocer la disposición de las citadas variables por parte de las empresas. Se contrasta si hay asociación entre la tipología de capital de la empresa y los atributos seleccionados, así como entre éstos y el tamaño de la empresa. Por último, se seleccionan las empresas que realizan innovaciones de productos y se realiza un análisis multivariante utilizando la técnica Homals para determinar las variables nominales más relacionadas entre sí, y con las puntuaciones objeto obtenidas contrastar si hay diferencias significativas según la nacionalidad y el tamaño empresarial.

Se verifica que las grandes empresas son las que realizan más I+D+i e incorporan los sistemas y técnicas objeto de estudio. En cambio, las pequeñas son las que menos, estando las pequeñas de capital nacional en una desventaja competitiva en el sector.

\section{Innovación tecnológica. Sistemas y técnicas para el diseño y desarrollo de nuevos productos}

La competencia actual en el sector exige que las empresas suministren productos de mayor valor añadido y contenido tecnológico, necesitando realizar más
I+D+i. El término I+D+i engloba 3 actividades según el Manual de Frascati (OECD 2002):

Investigación aplicada. Consiste en trabajos originales realizados para adquirir nuevos conocimientos; que a diferencia de la investigación básica, está dirigida fundamentalmente hacia un objetivo práctico específico.

Desarrollo experimental. Consiste en trabajos sistemáticos que aprovechan los conocimientos existentes obtenidos de la investigación y/o la experiencia práctica, que está dirigido a la producción de nuevos materiales, productos o dispositivos; a la puesta en marcha de nuevos procesos, sistemas y servicios, o a la mejora sustancial de los ya existentes.

La innovación es la transformación de una idea en un producto vendible nuevo o mejorado o en un proceso operativo en la industria.

Las innovaciones tecnológicas constan de la innovación en producto y la innovación en procesos. La OECD (2005) en el Manual de Oslo establece las siguientes definiciones:

- La innovación en producto es la introducción de nuevos bienes/servicios o mejoras significativas en las características o aplicaciones de los ya existentes.

- La innovación en proceso es la implantación de nuevos métodos de producción y entrega o mejoras significativas en los ya existentes.

En el proceso de diseño y desarrollo se materializa la innovación del producto.

La diferenciación entre diseño ${ }^{2}$ y desarrollo es confusa en gran parte de la literatura, incluso ciertos autores no los diferencian (Osteras et al., 2006). Para Montaña (1989) el diseño del producto va desde la fase posterior a concebir la idea hasta la etapa previa al prototipo. Osteras et al. (op. cit.) consideran que al diseño le corresponden los niveles del diseño conceptual y el diseño de detalle del componente, mientras que la fase del desarrollo incorpora los ni-

\footnotetext{
I Las empresas de capital extranjero suelen ofertar productos de mayor valor añadido y contenido tecnológico En cambio, las de capital nacional suelen tener productos de inferior valor añadido y contenido tecnológico.

La incidencia del tamaño empresarial en la innovación ha generado cierta controversia en la literatura empresarial, donde Camisón et al. (2002), tras aplicar un meta análisis a artículos de prestigio, llegan a la conclusión de que la correlación tamaño-innovación es significativa y positiva, si bien no intensa.

2 Para Montaña y Moll (2006) diseñar es definir las características estructurales, fisonómicas y funcionales necesarias para que un producto pueda materializarse y cumplir su misión.
} 
veles del desarrollo del componente y el desarrollo del prototipo.

El grado de implicación de los proveedores en el diseño y desarrollo de nuevos productos viene condicionado por el rol que le deja desempeñar el fabricante en las diferentes fases de $1+D+i$ de un nuevo modelo ${ }^{3}$.

Las empresas para ser competitivas deben gestionar unos procesos de desarrollo que ofrezcan nuevos productos más flexibles y eficientes (Muffatto, 1999), necesitando incorporar sistemas y técnicas que ofrezcan menor time to market, mayor calidad y menores costes en el diseño y desarrollo de nuevos productos. Se han seleccionado un conjunto de las mismas para este estudio, que a continuación comentamos. La ingeniería simultánea es un sistema organizativo del proceso de diseño y desarrollo que se caracteriza por constituir un equipo multifuncional, generalmente bajo una organización matricial, implicándose en la planificación, organización, dirección y control de todas las actividades relacionadas con productos y procesos, desde la generación de la idea hasta la terminación del producto, realizando en paralelo el mayor número de tareas (Clark y Fujiomoto, I99 I; Jürgens, 200 I). Requiere considerar la importancia de colaborar con clientes y proveedores (Martínez y Pérez, op cit.), ayudando en una mejor especificación del producto y mayor adecuación de los materiales y piezas, así como poder disponer y absorber más conocimiento externo útil, siendo conveniente también cooperar con otros agentes externos que lo ofrezcan (incorporando la open innovation, en terminología de Chesbrough, 2003). Las empresas que adoptan la ingeniería simultánea consiguen grandes beneficios en términos de reducir el time to market, los costes de desarrollo e impulsar la calidad (Sapuan et al., 2006).

Para Barba (2003), Aguayo y Soltero (op. cit.) y Sapuan et al (op. cit.) la ingeniería simultánea requiere tener el soporte de determinadas técnicas de diseño como son el AMFE, el QFD, el Análisis del valor, la Ingeniería del valor, los métodos Taguchi/DOE y el DFMA, así como los sistemas de información CAD, CAE, CAM y prototipado rápido, ayudando a reducir el time to market, mejorar la calidad, bajar los cos- tes y obtener más productividad en la I+D+i (Droge et al., 2000; Martínez y Pérez, op. cit.).

El CAD/CAE acelera los procesos de diseño y cambios posteriores de ingeniería (Martínez y Pérez, op. cit.), posibilitando simular condiciones de funcionamiento reales. El uso integral del CAD/CAM/CAE, presupone que los datos están en constante intercambio entre los diferentes actores implicados en el desarrollo de nuevos productos, que crean una red entre ellos (Perunovic y Christiansen, 2005), reduciendo el tiempo de desarrollo, los costes y mejorar la calidad (Lin y Kuo, 2008). La necesidad de reducir el coste del prototipo físico, así como el ciclo de diseño y desarrollo exige utilizar también tecnologías de prototipado rápido, para construir prototipos en pocas horas a partir de los datos que facilita el CAD/CAE (Miranda et al., op. cit.), mejorando la eficiencia y fiabilidad del diseño del producto (Guangchun et al., 2004).

El AMFE incorpora la voz del ingeniero, mientras que el QFD recoge la voz del cliente (Ginn et al., 1998). Mediante el AMFE se analizan los riesgos de los proyectos de ingeniería, anticipando los problemas potenciales del producto (Puente et al., 2002). Lager (2005) realizó un metaanálisis obteniendo que el QFD favorece el obtener mejores productos y diseños, la satisfacción del cliente y que la información se disemine en la organización. En cambio, no hay evidencia científica de que reduzca el time to market. Los Métodos Taguchi/DOE posibilitan obtener un diseño robusto y se incorporan como técnica para buscar soluciones conceptuales a los ¿cómo? de las matrices QFD. He et al. (2006) han comprobado que se obtienen mejores resultados al utilizar de forma integrada las herramientas de calidad, como el QFD, AMFE y DOE La integración del QFD y el Análisis Valor permite anticipar los intereses del cliente y obtener un producto que cumpla con su función al menor coste posible, identificando componentes o fases del producto sin valor añadido (Martínez y Pérez, op. cit). La aplicación del Análisis valor durante el diseño se denomina Ingeniería del valor (Fernández et al., 2006). Por otra parte, la integración del QFD, Análisis valor y el DFMA, permite optimizar el diseño, puesto que el QFD y el Análisis Valor evalúan el impacto de las modificaciones que sugiere el DFMA

\footnotetext{
3 Se establece la siguiente tipología (Clark y Fujimoto, 1991; Caputo y Zirpoli, 2002; Volpato 2004):

- El proveedor realiza desde el concepto del componente hasta su fabricación.

- Los proveedores ejecutan el diseño detallado basado en las especificaciones dadas por el fabricante.

- Codiseño entre fabricante y proveedores.

- El diseño del componente lo realiza el fabricante y luego coge los proveedores a través de licitaciones para que se responsabilicen de la ingeniería de proceso y la producción. (Hsuan, 2003).
} 
(Mendoza el al., 2003). Yeh et al. (2008) asimismo verifican que el DOE, AMFE y la implicación de los proveedores influyen positivamente en el desarrollo de nuevos productos y en diversos indicadores de perfomance.

La participación de los proveedores en el desarrollo de nuevos productos, tal como indica Hsuan (2003) reduce el tiempo de duración del proyecto, los costes del proyecto, y mejora la calidad percibida. Si bien no siempre la colaboración con los proveedores es beneficiosa (Wynstra et al., 2001; Langner y Seidel, 2009).

Para la industria auxiliar española Martínez y Pérez (op. cit.) encontraron que la ventaja percibida en costes de introducción estaba correlacionada con el DFM, prototipado rápido y análisis valor. En cambio, la ventaja percibida en costes de desarrollo se correlaciona más con el DFM, la asociación con proveedores y el prototipado rápido.

\section{Metodología}

Se analizan los datos de una encuesta realizada a una muestra significativa de empresas, utilizando determinadas técnicas estadísticas, con la finalidad de conocer los objetivos que se comentan a continuación.

\section{I. Estructura y objetivos del cuestionario}

El cuestionario diseñado y enviado a las empresas tiene el objetivo de analizar en el colectivo objeto de estudio las siguientes variables:

- $\mathrm{N}^{\circ}$ de personas en I+D+i, como aproximación de la capacidad de I+D+i de las firmas.

- Si efectúan innovación de productos (comprobando si realizan las fases Investigación, Diseño y Desarrollo del producto) e innovación de procesos.

- Si utilizan los sistemas de automatización: CAD, CAM, CAE, Rapid prototiping.

- Si aplican como métodos y técnicas para gestionar el diseño y desarrollo de nuevos productos: ingeniería simultánea, análisis valor, ingeniería valor, AMFE, DFMA, QFD, Métodos Taguchi/DOE, colaborar con clientes y proveedores.

\subsection{Análisis estadístico a efectuar}

Para la variable cuantitativa «Personal asignado a I+D+i», tras segmentar la muestra según la nacionalidad del capital y el tamaño empresarial, se calculan para cada distribución de frecuencias las medidas de síntesis de posición central y no central, así como de dispersión. Posteriormente se contrastan siguientes las hipótesis nulas sobre tal variable:

HI: Igualdad en la medida de tendencia central según la nacionalidad del capital

H2: Igualdad en la medida de tendencia central según el tamaño empresarial

Se aplican respectivamente los contrastes de $U$ de Mann Whitney y Kruskal y Wallis. En caso de rechazarse, los rangos promedios permiten determinar en cada caso la categoría donde el personal de $1+\mathrm{D}+\mathrm{i}$ es superior.

Para los atributos (variables dicotómicas que recogen la presencia o ausencia de la característica de interés) se elaboran las respectivas distribuciones univariantes, donde las proporciones muestrales permiten conocer la mayor presencia de tales atributos según el capital y el tamaño empresarial. Posteriormente se contrastan las siguientes hipótesis nulas:

H3: Independencia de cada atributo individual con la variable nacionalidad del capital

H4: Independencia de cada atributo individual con la variable tamaño empresarial

Se aplican el test chi cuadrado de Pearson o bien el test exacto de Fisher (si la frecuencia absoluta esperada es inferior a 5): Cuando se rechaza la hipótesis $\mathrm{H} 3$ el grado de asociación de cada atributo con la variable nacionalidad se obtiene con los coeficientes de contingencia ${ }^{4}$ y $Q$ de Yule ${ }^{5}$. En caso de rechazarse $\mathrm{H} 4$ el estadístico para la asociación de cada atributo y el tamaño empresarial es la $\vee$ de Crámer ${ }^{6}$.

\footnotetext{
4 Toma el valor cero si no hay asociación y tiende a uno cuanto mayor es la asociación, aunque no llega al mismo.

5 Es una medida de asociación global e indica la dirección de la asociación, con rango de variación entre - I y I. Como medida de asociación en tablas 2*2 son más apropiados los valores de la Q de Yule (Ato y López, 1996).

6 Toma el valor cero si no hay asociación y tiende a uno cuanto mayor es la asociación.
} 
Se analiza también para el ejercicio del año 2003 si hay diferencias significativas en la medida de tendencia central de los ratios Resultado de explotación/lmporte neto ventas (H5) y Resultados de explotación/Activo total (H6), según realice la empresa respectivamente «Innovación de productos» e «lnnovación de productos para obtener nuevos productos», para el conjunto de empresas, así como segmentando por nacionalidad y por tamaño empresarial.

Posteriormente, de la elaboración de sucesivas tablas de contingencia múltiples se determina la proporción de empresas que aplican conjuntamente diferentes combinaciones de los sistemas, técnicas y métodos considerados, verificándose en qué modalidades hay mayor presencia conjunta. Finalmente, en las empresas que hacen innovación de productos mediante el análisis Homals se busca describir las relaciones de más de dos variables nominales en un espacio de pocas dimensiones, e identificar grupos de técnicas y sistemas más relacionadas entre sí y con qué fases de I+D. Permite cuantificar simultáneamente diversas variables nominales, obteniendo los puntos objetos, sobre los que aplicar los contrastes $U$ de Mann Whitney y Kruskal y Wallis, para contrastar si hay diferencias significativas en la medida de posición central de los puntos objeto según la nacionalidad del capital o el tamaño empresarial (hipótesis $\mathrm{H7}$ y H8).

\subsection{Ficha técnica de la encuesta}

La encuesta se ha realizado en Cataluña, adoptando como población las empresas proveedoras de los fabricantes de automóviles $(N=1 \mid 5)$, seleccionando una muestra final de 100 empresas. Los elementos de muestreo han sido los directores de los departamentos de I+D o bien de ingeniería, a los que se realizó una encuesta telefónica y/o vía mail. El período temporal en que se efectuó la encuesta fue el año
2003, verificando posteriormente que contestaron correctamente durante el año 2007.

Considerando la estimación de las proporciones poblacionales y el método de la máxima holgura (proporción poblacional $=0,5)$, el margen de error de muestreo de la estimación es del 3,8 \%.

\subsection{Características de la muestra}

El $29 \%$ de las empresas son de capital nacional y el $71 \%$ de capital extranjero (tabla I). Hay una importante presencia de las grandes empresas (casi el 50\% tienen más de 250 trabajadores), mayoritariamente de capital extranjero.

Tabla I

Distribución del total empresas según el tamaño ( $\mathrm{n}^{\circ}$ trabajadores) y la nacionalidad

\begin{tabular}{|l|c|c|c|c|c|}
\hline & $\begin{array}{c}\text { Hasta } \\
\mathbf{1 0 0}\end{array}$ & $\mathbf{I 0 I - 2 5 0}$ & $\mathbf{2 5} \mathbf{I - 5 0 0}$ & $\begin{array}{c}\text { Mayor } \\
\mathbf{5 0 0}\end{array}$ & Total \\
\hline Española & 9 & 7 & 6 & 7 & 29 \\
\hline Extranjera & 9 & 26 & 15 & 21 & 71 \\
\hline Total & 18 & 33 & 21 & 28 & 100 \\
\hline
\end{tabular}

\section{Análisis de las variables. Explotación estadística}

\section{I. Personal asignado a I+D}

Uno de los indicadores utilizados para conocer la capacidad de las empresas de efectuar $1+D+i$ es la variable "Personal que realiza actividades de I+D+i". El elevado coeficiente de variación en las distribucio-

Tabla 2

Personal en I+D+i. Segmentación según nacionalidad del capital y tamaño empresarial.

\section{Principales estadísticos descriptivos}

\begin{tabular}{|l|c|c|c|c|c|c|}
\hline \multicolumn{1}{|c|}{ Variables } & Media & C.v. (\%) & Media recortada al 5\% & Primer cuartil & Mediana & Tercer cuartil \\
\hline Capital nacional & 13,1 & 262 & 7,0 & 0 & 5 & 12,0 \\
\hline Capital extranjero & 11,4 & 247 & 6,2 & 0 & 4 & 10,0 \\
\hline Hasta I00 trabajadores & 1,2 & 150 & 1,1 & 0 & 0 & 2,3 \\
\hline I0I-250 trabajadores & 5 & 120 & 4,4 & 0 & 3 & 9 \\
\hline 25।-500 trabajadores & 6 & 110 & 5,0 & 1 & 5 & 8 \\
\hline$>500$ trabajadores & 31,7 & 164 & 24,3 & 0,75 & 16,5 & 28,5 \\
\hline
\end{tabular}


nes de las diferentes modalidades consideradas (tabla 2), implica que las medidas representativas de tendencia central sean la media recortada y la mediana, y no la media.

Las empresas de capital nacional ofrecen valores algo superiores en las medidas de posición central de la variable «Personal en I+D+i tegoría «Hasta 100 trabajadores» tiene mediana nula y un tercer cuartil bastante más reducido al resto, donde destacan las de más de 500 trabajadores por tener más capacidad de hacer $1+D+i$, con valores claramente superiores en la mediana y tercer cuartil.

El contraste $U$ de Mann Whitney refleja que no hay diferencias significativas en la medida de tendencia central según la nacionalidad del capital $(U=953,5$ y p-valor $=0,556)$. En cambio, sí se observan según el tamaño empresarial, con el contraste de Kruskal y Wallis (chi cuadrado $=20,4$ y $p$-valor $=0,000$ ), y los rangos promedios (tabla 4) ofrecen una relación directa respecto las categorías de tamaño empresarial. Ello es consecuencia de que la mayoría de pequeñas empresas de capital nacional y Pymes de capital extranjero ubicadas en Cataluña no realizan I+D. En las Pymes de capital extranjero sus grupos suelen realizar en el extranjero las innovaciones de producto que se incorporan en los productos que fabrican en España. En cambio, las de más de 500 trabajadores son las que hacen más I+D+i en Cataluña.

Tabla 3

Rangos promedios del contraste de Kruskal y Wallis segmentando según tamaño empresarial

\begin{tabular}{|c|c|c|c|c|}
\hline & $\begin{array}{c}\text { Hasta } \\
\mathbf{I 0 0}\end{array}$ & $\mathbf{I 0 I - 2 5 0}$ & $\mathbf{2 5} \mathbf{I - 5 0 0}$ & $\mathbf{>} \mathbf{5 0 0}$ \\
\hline Rango promedio & 29,7 & $46, \mathbf{I}$ & 52,8 & 67,3 \\
\hline
\end{tabular}

\subsection{Innovación tecnológica, sistemas y métodos para el diseño y desarrollo de productos}

\subsubsection{Análisis univariante y bivariante}

En las tablas 4 y 5 se presentan los porcentajes de empresas que disponen cada atributo individual, diferenciando según la nacionalidad del capital y el tamaño empresarial. En las multinacionales de capital extranjero la información es sólo de las filiales ubicadas en Cataluña. De los resultados destacamos que la mayoría de capital nacional (68\%) y extranjero (57\%) afirman realizar innovaciones de productos.
Aproximadamente tres de cada cuatro grandes empresas realizan innovaciones de productos (todas las grandes de capital nacional y la mayoría de capital extranjero), por sólo una de cada tres pequeñas. Casi el doble de las empresas nacionales respecto las de capital extranjero hacen «lnvestigación» (54\% frente a un $29 \% \cdot$ ), siendo las grandes empresas quienes más realizan tal actividad. Algo más de la mitad de las empresas de capital nacional hacen «Diseño» (53\%), siendo inferior en las de capital extranjero $(41,7 \%)$. Asimismo, más de la mitad efectúan «Desarrollo de productos», superando las de capital nacional también casi en diez puntos a las de capital extranjero (67,9\% frente al 58,3\%, respectivamente). Se debe a que las empresas de capital extranjero que sólo fabrican en España (41,7\%), el diseño y desarrollo de sus productos se realizan en la matriz u otra filial exterior.

En las empresas de capital extranjero el 16,6\% hacen «Desarrollo» pero no «Diseño» (les viene dado desde otras partes del grupo), efectuando en Cataluña el prototipo, las homologaciones y en mayor medida la ingeniería de fabricación. Asimismo, el 14,3\% de las empresas de capital nacional no hacen diseño del producto pero sí desarrollo de producto, al venirles dadas las especificaciones y planos por el fabricante, acabando el proveedor de desarrollar la pieza. Realizan el prototipo del producto, las muestras iniciales y ensayos correspondientes, trabajando generalmente en codesarrollo con el fabricante. Estas empresas adaptan el producto del cliente, aplicando su tecnología.

Se verifica una elevada proporción de empresas que «lnnovan en procesos», siendo total en las grandes (ver tabla 6), al necesitar optimizar sus procesos para ajustarse a las reducciones de precios de los fabricantes, buscando continuas eficiencias y mejoras en su productividad, mayor flexibilidad y capacidad productiva.

Las pequeñas empresas son las que hacen menos «Diseño» y «Desarrollo de producto», no efectuando ninguna de ellas «Investigación», incidiendo que las pequeñas de capital nacional ofrecen productos de menor valor añadido. En cambio, las grandes son las que más efectúan tales fases, suministrando generalmente sistemas o módulos, de mayor complejidad tecnológica. Disponen de más recursos para realizar $1+D+i$, tienen mayor intensidad de innovación, y obtienen superiores economías de escala que les permiten recuperar antes sus gastos en $1+D+i$. Estas empresas colaboran con el fabricante en fases más tempranas del diseño de nuevos mo- 
delos, como socios estratégicos, y también colaboran más con sus proveedores buscando conocimientos externos y complementariedades tecnológicas, creando redes.

Seleccionando 98 empresas disponibles en la muestra en la base $S A B I$, se contrasta si hay diferencias significativas en la medida de posición central de los ratios Rdo. de explotación/lmporte neto cifra de negocios y Rdo. de explotación/Activo Total según realicen o no «Innovación de productos», o bien «lnnovación de productos obteniendo nuevos productos», resultando afirmativo para esta segunda variable, con $p$-valor de 0,011 y 0,03 , ofreciendo rangos promedios mayores en las que se realiza. Si segmentamos por nacionalidad, en las de capital extranjero se verifica también para la «lnnovación de productos obteniendo nuevos productos» ( $p$-valor $=0,048$ y 0,017 respectivamente), con rangos promedios superior en las que hacen tal tipo de innovación. Sin embargo, en las de capital nacional no hay tal diferencia significativa ( $p$-valor $=0,137$ y 0,094), posiblemente porque quienes la hacen han necesitado asumir mayores costes por su internacionalización, logística, e inversiones en maquinaria para ajustarse a las demandas de los fabricantes, afectándoles a sus cuentas de resultados.

Si analizamos los sistemas de automatización verificamos la alta disposición del «CAD». En cambio, el «CAD/CAM» y el «CAE» lo utilizan una proporción media del total de empresas de capital nacional, así como de las grandes empresas (57, I\%). En los sistemas «CAD», «CAD/CAM» y «CAE» hay una relación directa entre la proporción muestral de empresas que lo utilizan y el tamaño empresarial. Ello queda más reflejado en el «Prototipado rápido», que sólo lo incorpora una empresa de las pequeñas. Por tanto, de éstas casi todas carecen de los sistemas tecnológicos para realizar actividades de diseño/desarrollo de manera eficaz y eficiente. En cambio, el 57, I\% del total de las grandes (el $94 \%$ de las grandes que hacen Diseño), utilizan el «CAD/CAM», «CAE» y el «Prototipado rápido», por estar desde las fases iniciales del diseño del nuevo modelo del fabricante, $y$ deber de ajustarse a los requerimientos de los clientes en calidad, coste y plazo en el diseño de nuevos productos. Su utilización les ayuda a seguir optando para ser seleccionado como proveedor por su capacidad tecnológica en futuros modelos.

Al analizar según la nacionalidad del capital (véase tabla 4), las frecuencias relativas maestrales ofrecen diferencias al menos de diez puntos porcentuales a favor de las de capital nacional en los sistemas
«CAD», «CAE»; «CAD/CAM» y en la «Ingeniería simultánea» debido a que algunas de las extranjeras hacen en Cataluña menos fases de diseño y desarrollo, por lo que las precisan menos.

En casi todas las técnicas consideradas hay una relación directa entre las proporciones muestrales de empresas y el tamaño empresarial. En las pequeñas empresas ninguna aplica el «QFD», los «métodos Taguchi/DOE» y la «lngeniería del valor», porque no suelen participar en el diseño de la pieza, los directivos no le dan la relevancia que tienen, y carecen de personal con conocimiento en las dos primeras. Además, el QFD requiere importantes esfuerzos y disciplina en su aplicación, consumiendo bastante tiempo (Thia et al., 2005), dificultando más su implantación en las pequeñas empresas.

Según la nacionalidad del capital, en ninguna de las categorías se supera que la mitad de las empresas utilicen individualmente el «Prototipado rápido», el «QFD», la «lngeniería del valor», o bien los «Métodos Taguchi/DOE». Las técnicas «QFD», «Métodos Taguchi» e «Ingeniería del valor» se incorporan en mayor proporción en las extranjeras, porque las que hacen diseño en España participan en mayor medida en etapas más tempranas del diseño, disponen de personal preparado en tales técnicas, y se incorporan también en sus matrices dándose un efecto imitación en la filial española. Miranda y Bañeguil (2002) señalan que la cultura de las direcciones empresariales en España es no utilizar tales técnicas, puesto que suponen cambios en las organizaciones y procesos. De los resultados de este estudio es difícil sustentar tal afirmación para las grandes empresas. En cambio, sí es válido para las pequeñas que deberían tratar de aplicarlas, dándole la importancia que tienen, $y$ tener personal cualificado para su implementación.

Al contrastar la hipótesis nula de independencia entre la variable nacionalidad del capital respecto cada atributo, sólo para la variable «Investigación» se rechaza la hipótesis nula de independencia con la variable nacionalidad del capital. En tal caso el coeficiente phi es de $-0,223$ y el $Q$ de Yule $=-0,47$ (intensidad de asociación media).

Se verifica la relación directa entre la frecuencia relativa muestral de empresas que dicen realizar «lnvestigación», o bien efectuar «Innovación para obtener nuevos productos» con el tamaño empresarial (tabla 4). Como medida de asociación entre cada atributo y el tamaño empresarial es la $\vee$ de Crámer, mayoritariamente de grados medio-bajos. 
Tabla 4

Tipos de innovación tecnológica efectuada, fases I+D (a) sistemas de automatización y técnicas utilizadas, según tamaño empresarial para el total de empresas. Frecuencias. Contraste de independencia entre cada ítem y la variable tamaño empresarial.Valor del estadístico V de Cramer.

\begin{tabular}{|c|c|c|c|c|c|c|c|c|c|}
\hline & $\begin{array}{c}\text { Nacional } \\
(\%)\end{array}$ & $\begin{array}{c}\text { Extranjero } \\
(\%)\end{array}$ & $\begin{array}{c}\text { Hasta } \\
100(\%)\end{array}$ & $\begin{array}{c}101-250 \\
(\%)\end{array}$ & $\begin{array}{c}251-500 \\
(\%)\end{array}$ & $\begin{array}{l}>500 \\
(\%)\end{array}$ & \begin{tabular}{|c|} 
Chi \\
cuadrado \\
(b)
\end{tabular} & $\begin{array}{l}\text { p-valor } \\
\text { (b) }\end{array}$ & $\begin{array}{l}\mathbf{V} \text { de } \\
\text { Crámer } \\
\text { (b) }\end{array}$ \\
\hline \begin{tabular}{|l} 
Innovación de \\
productos (c)
\end{tabular} & 67.9 & 58,3 & 38,9 & 57,6 & 76,2 & 67,9 & 6,45 & 0,09 & 0,26 \\
\hline \begin{tabular}{|l} 
Innovación de \\
productos (d)
\end{tabular} & 50,0 & 44,4 & 16,7 & 45,5 & 52,4 & 60,7 & 9,02 & 0,029 & 0,3 \\
\hline Investigación & 53,6 & 29,2 & 0,0 & 27,3 & 52,4 & 57,1 & 19,09 & 0,000 & 0,44 \\
\hline Diseño & 53,6 & 41,7 & $\mid 1,1$ & 45,5 & 52,4 & 60,7 & $|1,6|$ & 0,009 & 0,34 \\
\hline \begin{tabular}{|l|} 
Desarrollo \\
de productos
\end{tabular} & 67,9 & 58,3 & 38,9 & 57,6 & 76,2 & 67,9 & 6,45 & 0,92 & 0,25 \\
\hline Innovación en procesos & 89,3 & 95,8 & 77,8 & 93,9 & 100,0 & 100,0 & 11,5 & 0,009 & 0,34 \\
\hline CAD & 92,9 & 87,5 & 77,8 & 87,9 & 90,5 & 96,4 & 3,98 & 0,263 & 0,20 \\
\hline CAD/CAM & 57,1 & 37,5 & 22,2 & 33,3 & 57,1 & 57,1 & 8,43 & 0,038 & 0,29 \\
\hline CAE & 50,0 & 34,7 & 16,7 & 27,3 & 52,4 & 57,1 & 11,13 & 0,011 & 0,33 \\
\hline Prototipado rápido & 32,1 & 38,9 & 5,6 & 39,4 & 33,3 & 57,1 & 12,7 & 0,005 & 0,36 \\
\hline Ingeniería simultánea & 64,3 & 50,0 & 33,3 & 54,5 & 66,7 & 64,3 & 5,5 & 0,14 & 0,23 \\
\hline $\begin{array}{l}\text { QFD en } \\
\text { diseño/desarrollo }\end{array}$ & 28,6 & 33,3 & 0,0 & 30,3 & 38,1 & 50,0 & 13,0 & 0,005 & 0,36 \\
\hline QFD & 28,6 & 36,1 & 0,0 & 30,3 & 42,9 & 53,6 & 14,9 & 0,002 & 0,38 \\
\hline AMFE de producto & 57,1 & 51,4 & 16,7 & 51,5 & 66,7 & 67,9 & 14,8 & 0,002 & 0,39 \\
\hline Análisis de valor & 60,7 & 55,6 & 22,2 & 57,6 & 76,2 & 64,3 & 13,1 & 0,005 & 0,36 \\
\hline Ingeniería del valor & 14,3 & 30,6 & 0,0 & 21,2 & 28,6 & 46,4 & 16,7 & 0,005 & 0,36 \\
\hline \begin{tabular}{|l|}
$\begin{array}{l}\text { Mét.Taguchi/DOE } \\
\text { diseño/desarr. }\end{array}$ \\
\end{tabular} & 17,9 & 26,4 & 0,0 & 18,2 & 28,6 & 42,9 & 15,6 & 0,007 & 0,35 \\
\hline Métodos Taguchi/DOE & 39,3 & 44,4 & 0,0 & 39,4 & 57,1 & 64,3 & 20,7 & 0,000 & 0,45 \\
\hline DFMA & 39,3 & 40,3 & $\mid 1,1$ & 30,3 & 52,4 & 60,7 & 13,9 & 0,003 & 0,37 \\
\hline $\begin{array}{l}\text { Colaborar con clientes } \\
\text { y proveedores en la } \\
\text { I+D }\end{array}$ & 50,0 & 45,5 & 22,2 & 57,6 & 61,9 & 64,3 & 9,2 & 0,027 & 0,29 \\
\hline $\begin{array}{l}\text { Tamaño en cada } \\
\text { submuestra }\end{array}$ & $\begin{array}{c}28 \\
(100 \%)\end{array}$ & $\begin{array}{c}72 \\
(100)\end{array}$ & $\begin{array}{c}18 \\
(100 \%)\end{array}$ & $\begin{array}{c}33 \\
(100 \%)\end{array}$ & $\begin{array}{c}21 \\
(100 \%)\end{array}$ & $\begin{array}{c}28 \\
(100 \%)\end{array}$ & & & \\
\hline
\end{tabular}

(a) Se recogen sólo las fases de investigación, diseño y desarrollo del producto

(b) A partir de la tabla de contingencia del atributo y el tamaño empresarial

(c) para obtener productos sensiblemente mejorados y/o productos nuevos

(d) para obtener productos nuevos 
Las Pymes de capital extranjero al realizar menos $1+D+i$ tienen mayor riesgo de ser deslocalizadas en un futuro, por la competencia de otras plantas del grupo ubicadas en países que disponen de bajo coste laboral.

\subsubsection{Tablas de contingencia múltiples, combinando diversos atributos}

Para que el diseño y desarrollo de nuevos productos sea más eficaz deben utilizarse de forma conjunta (integrada) los sistemas y técnicas considerados, al darse sinergias y complementariedades entre los mismos. En la tabla 5 se recogen las frecuencias conjuntas asociadas a diversas combinaciones de ítems referidos a tales sistemas y técnicas, observándose que incorporar el conjunto de atributos lo realizan un número reducido de empresas en las diferentes modalidades. Si se tienen en cuenta sólo las empresas que hacen diseño entonces tales porcentajes prácticamente se duplican, pero la proporción de empresas que incorporan la totalidad de atributos sigue siendo reducida (13,2\% para las de capital nacional y 16,5\% para las de capital extranjero). Por tanto, la mayoría de empresas que hacen diseño deben esforzarse en su incorporación conjunta para conseguir mejores prestaciones de tales sistemas y técnicas, disfrutado de las ventajas comentadas.

Tabla 5

Combinaciones de variables. Frecuencias relativas según nacionalidad o bien tamaño empresarial. Total empresas

\begin{tabular}{|c|c|c|c|c|c|c|}
\hline $\begin{array}{l}\text { Atributos incorporados } \\
\text { conjuntamente }\end{array}$ & Nacional & Extranjero & Hasta 100 & $\begin{array}{l}101-250 \\
(\%)\end{array}$ & $\begin{array}{c}251-500 \\
(\%)\end{array}$ & $>500(\%)$ \\
\hline CAD/CAE & 50 & 34,7 & 16,7 & 27,3 & 52,4 & 57,1 \\
\hline CAD/CAM/CAE & 46,4 & 26,4 & 16,7 & 24,2 & 42,9 & 50,0 \\
\hline CAD/CAM/CAE/Prototipo rápido & 28,6 & 22,2 & 0,0 & 21,2 & 19,0 & 46,4 \\
\hline $\begin{array}{l}\text { CAD/CAM/CAE/Prototipo rápido/ AMFE pro- } \\
\text { ducto }\end{array}$ & 28,6 & 20,8 & 0,0 & 18,2 & 19,0 & 46,4 \\
\hline $\begin{array}{l}\text { CAD/CAM/CAE/Prototipo rápido/ AMFE pro- } \\
\text { ducto/I.S./A.V }\end{array}$ & 25,0 & 20,8 & 0,0 & 15,2 & 19,0 & 46,4 \\
\hline $\begin{array}{l}\text { CAD/CAM/CAE/Prototipo rápido/AMFE } \\
\text { producto/I.S./A.V./QFD }\end{array}$ & 21,4 & 13,9 & 0,0 & 9,1 & 9,5 & 42,9 \\
\hline $\begin{array}{l}\text { CAD/CAM/CAE/Prototipo rápido/ AMFE } \\
\text { producto/I.S./A.V./QFD/DFMA }\end{array}$ & 17,9 & 11,9 & 0,0 & 9,1 & 9,5 & 42,9 \\
\hline $\begin{array}{l}\text { CAD/CAM/CAE/Prototipo rápido/ AMFE } \\
\text { producto/I.S./A.V./QFD/Taguchi }\end{array}$ & 14,3 & 8,3 & 0,0 & 6,1 & 9,5 & 28,6 \\
\hline $\begin{array}{l}\text { CAD/CAM/CAE/Prot. rápido/ AMFE } \\
\text { producto/I.S./A.V/QFD/Taguchi/DFMA }\end{array}$ & 14,3 & 6,9 & 0,0 & 6,1 & 4,8 & 25,0 \\
\hline $\begin{array}{l}\text { CAD/CAM/CAE/Prot. rápido//AMFE } \\
\text { producto/I.S./A.V.//QFD/Taguchi/Ing. del valor }\end{array}$ & 13,6 & 6,9 & 0,0 & 6,1 & 0,0 & 25,0 \\
\hline $\begin{array}{l}\text { CAD/CAM/CAE/Prot. rápido/AMFE } \\
\text { producto /I.S./A.V./QFD/Taguchi/ I.V.//DFMA }\end{array}$ & 7,1 & 6,9 & 0,0 & 6,1 & 0,0 & 17,9 \\
\hline $\begin{array}{l}\text { CAD/CAM/CAE/Prot. rápido/AMFE } \\
\text { producto /I..S./A.V./QFD/Taguchi/ I.V./DFMA/ } \\
\text { Colaborar con clientes y proveedores }\end{array}$ & 7,1 & 6,9 & 0,0 & 6,1 & 0,0 & 17,9 \\
\hline Tamaño submuestras (100\%) & $28(100 \%)$ & $72(100 \%)$ & $18(100 \%)$ & $33(100 \%)$ & $21(100 \%)$ & $28(100 \%)$ \\
\hline
\end{tabular}


Suele haber una relación directa entre la proporción muestral y el tamaño empresarial en bastantes de las diferentes combinaciones seleccionadas. Se verifica que pocas pequeñas empresas han integrado el CAD, CAM y CAE y ninguna también el prototipado rápido. Si le añadimos la escasa utilización del QFD y Métodos Taguchi/DOE entonces no tienen capacidad de realizar diseño y desarrollo de sus productos de manera eficiente y eficaz, por lo que las de capital nacional tendrán dificultades futuras de que el fabricante les seleccione ante la mayor concentración de sus proveedores. En cambio, están más incorporadas en las más grandes (superan los 500 trabajadores), utilizando todos los ítems un 18\% de las mismas (30\% si consideramos las más grandes que sólo hacen diseño), que corresponden a empresas que hacen actividades de investigación, diseño y desarrollo de producto. Colaboran más con los fabricantes, disponen de mayor personal dedicado a I+D+i, y sus direcciones apuestan decididamente por obtener nuevos productos, incorporando los sistemas y técnicas consideradas, permitiéndoles reducir sus costes, mejorar la calidad y minorar los ciclos de diseño y desarrollo de sus productos, ayudándole a aumentar su competitividad.

\subsubsection{Análisis HOMALS sobre las empresas que realizan innovación de productos}

A continuación se presentan los resultados de utilizar la técnica Homals sobre las empresas que realizan innovación de productos. De las diferentes combinaciones de variables posibles aplicando el Homals, analizando los autovalores y las medidas de discriminación, se seleccionan las variables de la tabla 7 . Sólo el autovalor de la primera dimensión supera el cociente: $1 / \mathrm{n}^{\circ}$ de variables, es decir, el valor $1 / 16$. El de la segunda es ligeramente inferior, pero se considera para hacer el mapa de posicionamiento de las empresas en dos dimensiones.

La primera dimensión viene explicada en mayor medida por las variables: «Diseño»; «Prototipado rápido»; «Investigación»; «Ingeniería del valor»; «Ingeniería simultánea». Sus medidas de discriminación superan el valor 0,4. En cambio, la dimensión segunda viene explicada básicamente por el «DFMA».

En la tabla 6 se disponen cuantificadas las categorías en las dimensiones I y 2 . El valor uno recoge ausencia del ítem correspondiente y el valor dos su disposición.
Tabla 6

Cuantificaciones de las categorías

\begin{tabular}{|c|c|c|c|}
\hline & & Dimensión I & Dimensión 2 \\
\hline \multirow{2}{*}{$\begin{array}{l}\text { Diseño del } \\
\text { producto }\end{array}$} & $N_{0}=1$ & 1.317 & $-0,467$ \\
\hline & Sí = 2 & $-0,468$ & 0,165 \\
\hline \multirow[t]{2}{*}{ Prototipo rápido } & $N_{0}=1$ & 0,948 & $-0,2 \mid 3$ \\
\hline & Sí = 2 & $-0,615$ & 0,138 \\
\hline \multirow[t]{2}{*}{ Investigación } & $N_{0}=1$ & 0,837 & $-0,026$ \\
\hline & Sí = 2 & $-0,581$ & 0,018 \\
\hline \multirow[t]{2}{*}{ Ingeniería del valor } & $N_{0}=1$ & 0,560 & 0,057 \\
\hline & Sí = I & $-0,754$ & $-0,076$ \\
\hline \multirow[t]{2}{*}{$\begin{array}{l}\text { Ingeniería } \\
\text { simultánea }\end{array}$} & $N_{0}=1$ & 1,639 & 0,248 \\
\hline & Sí = I & $-0,247$ & $-0,037$ \\
\hline \multirow{2}{*}{ Análisis del valor } & $N_{0}=1$ & $|, 27|$ & $-0,113$ \\
\hline & Sí = 2 & $-0,249$ & 0,022 \\
\hline \multirow{2}{*}{ DFMA } & $N_{0}=1$ & 0,400 & 1,188 \\
\hline & Sí $=2$ & $-0,242$ & $-0,719$ \\
\hline
\end{tabular}

A continuación el gráfico I da las coordenadas de los casos en el espacio solución de dos dimensiones. La distribución de los valores de las empresas no es homogénea.

Gráfico I.

Empresas en dimensiones I y 2

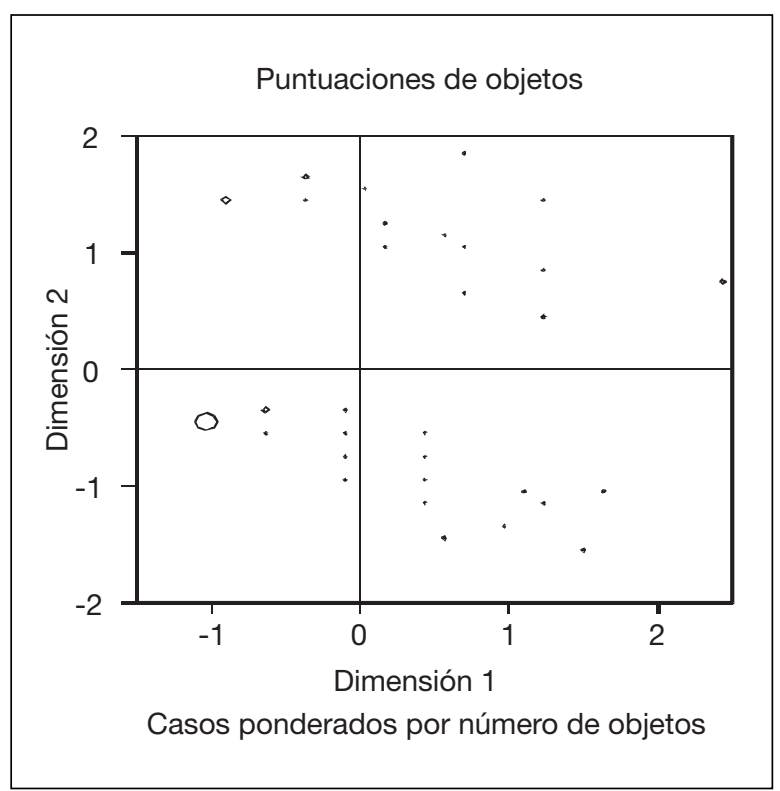


Se verifica que no existen diferencias significativas en la medida de posición central sobre los puntos objeto de la primera dimensión, segmentando según la nacionalidad de capital, utilizando la $U$ de MannWhitney $(U=357,5$ y p-valor $=0,5 \mathrm{I})$. En cambio, sí hay diferencias significativas según el tamaño empresarial aplicando el contraste de Kruskal y Wallis (valor chi cuadrado=21,9 y p-valor $=0,00$ ). Téngase en cuenta por la tabla 7 que las cuantificaciones de todos los ítems en la modalidad «Sí» tienen signo negativo; en cambio, la modalidad «No» son de signo positivo. Por tanto, debe interpretarse en sentido inverso el valor del rango promedio, es decir, las pequeñas que ofrecen mayor rango realmente (véase tabla 8) incorporan menos los ítems que discriminan la primera dimensión, en cambio las de más de 500 trabajadores, con menor rango, los incorporan más.

Tabla 7

Rangos promedios según tamaño empresarial en dimensión I

\begin{tabular}{|l|l|l|l|l|}
\hline \multicolumn{1}{|c|}{ Categorías } & $\mathbf{I}-\mathbf{~} \mathbf{0 0}$ & $\mathbf{I 0} \mathbf{I}-\mathbf{2 5 0}$ & $\mathbf{2 5} \mathbf{I - 5 0 0}$ & $\mathbf{>} \mathbf{5 0 0}$ \\
\hline Rango promedio & 54,57 & 32,26 & 33,53 & 18,92 \\
\hline Tamaño submuestras & 7 & 19 & 16 & 19 \\
\hline
\end{tabular}

Por otra parte, en la dimensión 2 se verifica que no hay diferencias significativas en la medida de tendencia central según la nacionalidad $(U=321,5$ y pvalor $=0,23$ ), ni por el tamaño empresarial (chi cuadrado es 3,6 y p-valor $=0,31$ ).

\section{Conclusiones}

La información muestral permite verificar que la gran mayoría de las empresas innovan en procesos (siendo total en las grandes), para optimizar sus procesos y conseguir mejorar su productividad y flexibilidad. Bastantes empresas efectúan «Innovaciones de productos», existiendo relevantes diferencias entre las grandes y las pequeñas empresas, a favor de las primeras. Las empresas de capital nacional realizan en mayor proporción «Innovaciones de producto», «Diseño» y «Desarrollo de producto», explicable principalmente porque la mayoría de Pymes de capital extranjero las efectúan en sus matrices u otras filiales en el extranjero, próximas a los centros técnicos de los fabricantes, en países donde hay más relación empresa-universidad-centros tecnológicos.

Las empresas que realizan «Innovación de productos para obtener nuevos productos» obtienen mejores resultados de explotación sobre el importe neto de ventas, así como en el ratio resultados de explotación sobre el activo total. Se mantiene al considerar sólo las de capital extranjero, pero ello no entre las empresas de capital nacional.

No se obtienen diferencias significativas en la medida de tendencia central del personal dedicado a I+D+i, según la nacionalidad del capital, lo que es preocupante para la competitividad de las empresas de capital nacional, puesto que la mayoría de capital extranjero disponen de relevantes departamentos de I+D en el exterior. En cambio, las grandes empresas disponen en Cataluña de más personal dedicado a $1+\mathrm{D}+\mathrm{i}$, al realizar la mayoría más actividades de investigación, diseño y desarrollo.

El CAD/CAM y el CAE lo utilizan más las empresas de capital nacional y las grandes empresas. Excepto en la ingeniería simultánea y el análisis valor, el resto de técnicas se utilizan más en las de capital extranjero, lo que es una debilidad de la mayoría de Pymes de nacionalidad española y deberían subsanarlo.

En la mayoría de ítems hay una relación directa entre el tamaño empresarial y la proporción de empresas que utilizan cada ítem, siendo la asociación entre el tamaño empresarial y los ítems considerados en su mayoría medio-bajos o bajos. En cambio, la nacionalidad de capital sólo ofrece asociación significativa con el ítem «lnvestigación», al realizar tal actividad en mayor medida las empresas nacionales, pues en la mayoría de empresas de capital extranjero sus grupos deciden que se realice en el exterior, especialmente en el caso de las Pymes.

Las grandes empresas utilizan más los sistemas y técnicas considerados, por lo que obtienen una gestión más eficiente y eficaz de su I+D+i. En cambio, las pequeñas empresas son las que menos, siendo nula su actividad en investigación, baja en diseño y desarrollo, teniendo también una reducida o nula implantación conjunta de los sistemas y técnicas analizadas.

El análisis Homals recoge que las variables más relacionadas (según la primera dimensión obtenida) son: Diseño del producto, Prototipado rápido, Investigación; Ingeniería del valor e Ingeniería simultánea. Se 
verifica que hay diferencias significativas en los puntos objeto de la primera dimensión del Homals, incorporándose más tales ítems en las grandes empresas y en las pequeñas donde menos.

Por tanto, las grandes empresas ubicadas en Cataluña están utilizando más y mejor tales sistemas y técnicas, beneficiándose en mayor media de las ventajas que ofrece su integración. En cambio, las pequeñas empresas nacionales que están más presionadas por las políticas de los fabricantes del global sourcing y con mayor competencia de los proveedores de países de bajo coste, deberían tratar de asumir realizar un mayor diseño y desarrollo de nuevos productos, implantando más los sistemas y técnicas comentadas, para ofrecer productos de más valor añadido que les ofrezcan mayor diferenciación y tener más posibilidades de ser seleccionadas por unos fabricantes que exigen a sus proveedores mayor capacidad tecnológica y unos requerimientos más estrictos de costes, calidad y plazos en el diseño y desarrollo de nuevos productos. En caso contrario acabarán posiblemente bajando de nivel en la estructura piramidal de la industria auxiliar y asumir más riesgo de no poder sobrevivir ante la mayor competitividad del sector.

\section{Bibliografía}

AGGERI, F.y SEGRESTIN, B. (2007). «Innovation and Project development: an impossible equation?. Lessons from an innovative automobile project development», $R+D$ Management, Vol.37, No I, pp. 37-47.

AGUAYO, F.y SOLTERO,V.M. (2005). Metodología del Diseño Industrial. Un enfoque desde la ingeniería concurrente. Ed. Ra-ma, Madrid.

ANDERSON, J.; OLIVER, N. y ANDERSON, J. (200I). «Collaborative new product development in a multi-customer contest: challenges in Western auto component suppliers», International Journal of Automotive Technology and Management, Vol. I, No. 2-3

ATO, M. y LÓPEZ, J.J. (1996). Análisis estadístico para datos categóricos, Universidad de Murcia

BAÑEGUIL, T. M. y MIRANDA, F. J. (200I). «El tiempo de mercado como factor determinante del éxito de nuevos productos: mito o realidad», Investigaciones Europeas de Dirección y Economía de la Empresa, Vol. 7, No. 2, pp. I09- 122.

BARBA, E. (2003). La excelencia en el proceso de desarrollo de nuevos productos. Ed. EADA, Barcelona
CAMISIÓN, C.; LAPIEDRA, R.; SEGARRA, M. y BORONAT, M. (2002). Meta-análisis de la relación entre tamaño de empresa e innovación. Documento Instituto Valenciano de Investigaciones Económicas WP-EC 2002- I 5

CAPUTO, M. y ZIRPOLI, F. (2002). «Supplier in automotive component design: outsourcing strategies and supply chain management», Internacional Journal of Technology Management, Vol. 23, No. I/2/3, pp. $129-150$

CHESBROUGH, H.W. (2003). Open innovation: The new imperative for creating and profiting from technology. Harvard Business School Publishing, Cambridge

CLARK, K. y FUJIMOTO,T. (I99I). Product development perfomance», Strategy, Organization and Management in the World Auto Industry, Harvard Business School Press, Cambridge M.A

DROGE, C. JAYARAM, J.; VICKERY, S. y CALANTONE, R. (2000). «The ability to minimise the timing of new product development and introduction an examination of antecedent factors in the North American automotive supplier industry», Journal of Product Innovation Management, Vol. 17, No. I, pp. 24-40

GINN, D.M.; JONES, D.V.; RABNEJAL, H. y ZAIRL (1998). «The QFD/FMEA interface», European Journal of Innovation Management, Vol. I, No. I, pp. 7-20

GUANGCHUN, E., HUIPING, L., YANJIN, G. y GUOQUN, Z. (2004). «A rapid design and manufacturing system for product development applications», Rapid Prototyping Journal, Vol. I0, No. 3, pp. 200-206

HAMEL, G. y PRAHALAD, C.K. ( 1 994). Competing for the future, Harvard Business School Press, Boston

HE, Z., ZOU, F., CUI, Q., QI, E., LIU, Z. (2006). «Continuous improvement through integration of quality tools», Chinese Journal of Mechanical Engineering, Vol. 19, No. I, pp. 72-75

HSUAN, J. (2003). «Modularity, component, outsourcing, and Inter-firm learning», $R+D$ Management, Vol. 33, No. 4, pp. 439-454

JÜRGENS, U. (200I). «Approaches towards Integrating Suppliers in Simultaneous Engineering Activities: The Case of Two German Auto Makers», International Journal of Automotive Technology and Management, Vol. I, No. I, 200 I, 6I-77.

KOUFTEROS, X., VONDEREMBSE, M., JAYARAM, J.: (2005). «Internal and external integration for product development: the contingency effects of un- 
certainty, equivocality, and platform strategy»). Decision Science, Vol. 36, No. I, pp. 97-131 (2005)

LAMING, R. (1993). Beyond Partnership. Strategies for innovation and lean supply, Prentice Hall, London

LANGNER, B. y SEIDEL, V.P. (2009). «Collaborative concept development using suppliers competitions: insights from the automotive industry», Journal of Engineering Technology Management, Vol. 26, pp. $\mid 1-14$.

LANGERAK, F.y HULTINK, E.J. (2006). «The impact of product innovativeness on the link between development speed and new product profitability», Journal of Product Innovation Management, Vol. 23, No. 3, pp. 203-214

LARGER, T. (2005). «The industrial usability of quality function deployment: a literature review and síntesis on a meta-level», $R+D$ Management, Vol. 35, No. 4, pp. 409-426

LIN, B.T. y KUO, C.C. (2008). «Application of an integrated CAD/CAE/CAM system for stamping dies for automobiles», International Journal of Avanced Manufacturign Technology, Vol. 35, No. 9- I0, Pages 1000- 1013

MARTÍNEZ, A. y PÉREZ, M. (2003a). «Desarrollo de nuevos productos, contenido tecnológico y cooperación. Industria auxiliar de automoción». Economía Industrial, No. 353, pp. I | 3-122

MARTÍNEZ, A. y PÉREZ, M. (2003b). «Flexibility in new product development: a survey of practices and its relationship with the product's technological complexity», Technovation,Vol. 23, No. 2, pp. 139145.

MENDOZA, N., AHUETT, H. y MOLINA, A., (2003). Case Studies in the Integration of QFD, VE and DFMA during the Product Design Stage. The Proceedings of the 9th International Conference of Concurrent Enterprising, Espoo, Finland, I6-I 8 June 2003

MIRANDA, F.J.y BAÑEGUIL,T.M. (2002). «The effect of new product development techniques on new product success in Spanish firms»y. Industrial Marketing Management, Vol. 3I, No. 3, pp. 26I-27।

MONTAÑA, J. (1989). Diseño y marketing de nuevos productos. La gestión de producto en la empresa industrial. Tesis doctoral. Escuela Superior de Ingenieros Industriales. Universidad Politécnica de Cataluña.

MONTAÑA, J. y MOLL, I. (2006). Desenvolupament de producte: la gestió del disseny, Generalitat de Catalunya, CIDEM.
MOWERY, D.C., OSLEY, J.E. y SILVERMAN, B.S. (1996). "Strategic alliances and interfirm knowledge transfer», Strategic Management Journal,Vol. 17, Winter, pp. 77-92

MUFFATTO, M. (1999). "Building new product development capabilities", International journal of vehicle design, Vol. 2 I, No. I, pp. I-7

NONAKA, I. yTAKEUCHI, H. (1995). The knowledge creating company: How Japanese companies create the dynamics of innovation, Oxford University, Oxford

OECD (2002). The Measurement of Scientific and Technological Activities Frascati Manual. Proposed Standard Practice for Surveys on Research and Experimental Development, OECD, Paris.

OECD (2005: The Measurement of Scientific and Technological Activities: Guidelines for Collecting and Interpreting Innovation Data: Oslo Manual, OECD, Paris

OSTERAS, T; MURTHY, D.N.; RAUSAND, M. (2006). «Product perfomance and specification in new product development», Journal of Engineering Design, Vol. 17, No. 2, pp. 177-192

PERUNOVIC, Z.y CHRISTIANSEN,T.B. (2005). «EXploring Danish innovative manufacturing perfomance», Technovation,Vol. 25, No. 9, pp. I 05 I - 1058

PUENTE, J., PINO, R., PRIORE, P.y FUENTE, D. (2002), «A decision support system for applying failure mode and effects analysis», The International Journal of Quality \& Reliability Management, Vol. 19, No. I, pp. 137-50.

SAPUAN, S.M.; OSMAN, M.R.y NUKMAN,Y. (2006). «State of the art of the concurrent engineering technique in the automotive industry)s. Journal of Engineering Design, Vol. 17, No. 2, pp. $143-157$

THIA, C.W., CHAI, K.H., BAULY, J., XIN,Y. (2005). «An exploratory study of the use of quality tools and techniques in product development», TQM Magazine, Vol. 17, No. 5, pp. 406-424

VDA (2009). Auto Anual Repport 2008

VOLPATO, G. (2004). The OEM-FTS relationship in automotive industry», International Journal of Automotive Technology and Management, Vol. 4, No. 2/3, pp. 166-197

VON CORSWANT, F. y FREDRIKSSON, P. (2002). «Sourcing trends in the car industry», International Journal of Operations \& Product Management, Vol. 22, No. 7, pp. 74I-758

WOGNUM, P.M.; FISSCHER, O.,WEENINK, S. (2002). «Balanced relationships: management of client-sup- 
plier relationships in product development», Technovation, Vol. 22, No. 6, pp. 34I-35 I

WOMACK, J., JONES, D. y ROOS, D. (1990). The machine that changed the world, Maxwell Macmillan, Internacional, Nueva York

YEH , T.M. PAI, F.Y.: YAN, Ch. (2008). "Performance improvement in new product development with effective tools and techniques adoption for hightech industries».
Quality and Quantity.

Disponible en línea: http://www.springerlink.com/content/wk86|76v4628248I/

WYNSTRA, F., VAN WEELE, A. y WEGGEMANN, M. (200I). «Managing Suplier Involvement in Product Development: three critical issues», European Management Journal, Vol. 19, No. 2, pp. 157166. 